\title{
The importance of cyclic structure for Labaditin on its antimicrobial activity against Staphylococcus aureus
}

\author{
Simone C. Barbosa ${ }^{a, *}$, Thatyane M. Nobre ${ }^{a}$, Diogo Volpati ${ }^{\mathrm{b}}$, Pietro Ciancaglini ${ }^{\mathrm{c}}$, \\ Eduardo M. Cilli ${ }^{\mathrm{d}}$, Esteban N. Lorenzón ${ }^{\mathrm{e}}$, Osvaldo N. Oliveira Jr ${ }^{\mathrm{a}}$ \\ a Instituto de Física de São Carlos, Universidade de São Paulo, São Carlos, SP, Brazil \\ ${ }^{\mathrm{b}}$ Department of Natural Sciences, Mid Sweden University, SE-851 70 Sundsvall, Sweden \\ c Departamento de Química, Faculdade de Filosofia, Ciências e Letras de Ribeirão Preto, Universidade de São Paulo, Ribeirão Preto, SP, Brazil \\ d Instituto de Química, Universidade Estadual Paulista, Araraquara, SP, Brazil \\ e Departamento de Bioquímica e Biologia Molecular, Instituto de Ciências Biológicas, Universidade Federal de Goiás, Brazil
}

\section{A R T I C L E I N F O}

\section{Article history:}

Received 8 July 2016

Received in revised form 4 September 2016

Accepted 11 September 2016

Available online 17 September 2016

\section{Keywords:}

Antimicrobial peptide

Cyclic peptides

Peptide nanotubes

Labaditin

Langmuir monolayers

PM-IRRAS

Large unilamellar vesicles

Staphylococcus aureus

\begin{abstract}
A B S T R A C T
Antimicrobial resistance has reached alarming levels in many countries, thus leading to a search for new classes of antibiotics, such as antimicrobial peptides whose activity is exerted by interacting specifically with the microorganism membrane. In this study, we investigate the molecular-level mechanism of action for Labaditin (Lo), a 10-amino acid residue cyclic peptide from Jatropha multifida with known bactericidal activity against Streptococcus mutans. We show that Lo is also effective against Staphylococcus aureus (S. aureus) but this does not apply to its linear analogue $\left(\mathrm{L}_{1}\right)$. Using polarization-modulated infrared reflection absorption spectroscopy (PM-IRRAS), we observed with that the secondary structure of Lo was preserved upon interacting with Langmuir monolayers from a phospholipid mixture mimicking $S$. aureus membrane, in contrast to $\mathrm{L}_{1}$. This structure preservation for the rigid, cyclic Lo is key for the selfassembly of peptide nanotubes that induce pore formation in large unilamellar vesicles (LUVs), according to permeability assays and dynamic light scattering measurements. In summary, the comparison between Labaditin (Lo) and its linear analogue $\mathrm{L}_{1}$ allowed us to infer that the bactericidal activity of Lo is more related to its interaction with the membrane. It does not require specific metabolic targets, which makes cyclic peptides promising for antibiotics without bacteria resistance.
\end{abstract}

(c) 2016 Elsevier B.V. All rights reserved.

\section{Introduction}

Staphylococcus aureus is a Gram-positive bacteria that can cause several human illnesses such as skin infection, pneumonia, meningitis, osteomyelitis, endocarditis, toxic shock syndrome (TSS) and sepsis [1]. S. aureus is also the major cause of deadly bacteremia, much more aggressive than caused by other pathogens [2]. The treatment of these diseases is fraught with problems owing to the development of bacterial resistance. A classical case is the methicillin-resistant Staphylococcus aureus (MRSA), a strain of Staphylococcus bacteria that became resistant to antibiotics used for treating ordinary infections. This calls for the discovery of new antibiotics whose mechanism of action differs from the conventional ones, normally related to specific metabolic targets [3].

\footnotetext{
* Corresponding author.

E-mail address: simonebarbsa@gmail.com (S.C. Barbosa).
}

Antimicrobial peptides (AMPs) are promising as the next generation of antibiotics $[4,5]$, for they act on bacterial membranes and other generalized targets, thus making bacterial resistance unlikely [6]. AMPs develop an immunological role in a variety of plant and animal species, protecting these organisms from pathogen invasion [7-9]. They are able to differentiate cell membranes from eukaryotic to prokaryotic organisms [10], mostly due to their being usually positively charged and binding preferentially to anionic membranes, such as bacterial surfaces $[11,12]$. The mechanism of action of linear AMPs is well documented, including several models of membrane permeabilization/disintegration. On the other hand, not much is known about cyclic peptides [13], whose antibiotic activity depends on their conformational restrictions [14] and higher proteolytic stability compared to their linear analogues [15].

Labaditin (Lo) is a small head-to-tail cyclic AMP(VWTVWGTIAG) from Jatropha multifida with high hydrophobic content. In contrast to most of AMPs, it has no charged residues [16,17]. It has been proven effective against Streptococcus mutans, while its linear analogue $\left(L_{1}\right)$ is not [17]. Also, no hemolytic effect was observed for 
concentrations up to $100 \mu \mathrm{M}$ of Labaditin, indicating high selectivity of this peptide to microorganisms cells [17]. In this study, we designed a series of experiments to explain why Lo - but not $\mathrm{L}_{1}$ - is active, for which we used $S$. aureus as the bacteria of reference. In addition to performing bactericidal activity tests, we investigated the interaction of Lo and $\mathrm{L}_{1}$ with cell membrane models represented by Langmuir monolayers. Motivation for membrane studies arose from the hypothesis that bactericidal activity is directly correlated with interaction with the cell membrane. For elucidating the mechanism of action of Lo we also resorted to leakage assays and dynamic light scattering performed in large unilamellar vesicles (LUVs) to probe membrane permeabilization.

\section{Materials and methods}

\subsection{MIC determination}

S. aureus cells (ATCC 25923) were cultured in LB (Luria-Bertani) broth without shaking, and at a late exponential phase $\left(\mathrm{OD}_{600}=1.0\right)$ the culture was diluted to $\mathrm{OD}_{600}$ of 0.1 and used as inoculum. Microdilution was performed using a series of $200 \mu \mathrm{L}$ of LB broth and containing two fold serial dilution of one of the synthetic peptides (Labaditin Lo or its linear analogue $\mathrm{L}_{1}$ ), which were prepared in the 96-well microtiter plates. Approximately $10^{4}$ cells from the inoculum as described above were inoculated. The plates were incubated for $18 \mathrm{~h}$ at $37^{\circ} \mathrm{C}$, and the tests were performed in triplicate.

\subsection{Langmuir monolayers}

The peptides Lo and $\mathrm{L}_{1}$ were obtained from Aminotech Research ( $>95 \%$ purity), while 1,2-dioleoyl-sn-glycero-3phosphatidylglycerol (DOPG) and 14:0 Cardiolipin (CL) were purchased from Avanti Polar Lipids. All the solutions were prepared using Millipore Direct-Q ultra pure apyrogenic water (resistivity of $18.2 \mathrm{M} \Omega \mathrm{cm}$ at $25^{\circ} \mathrm{C}$ ), and the reagents were of the highest commercially available purity grade. For producing Langmuir monolayers, we simulated the phospholipid composition of S. aureus with 55\% DOPG and 45\% CL, according to the literature [18-20], named from now on S. aureus PL. It is worth mentioning that we do not neglet the importance of lipid composition, with the presence of branched lipids for example, for the bacterial susceptibility against antimicrobial peptides [33]. However, we decided to focus our efforts on determining the role of peptideís cyclic structure on its interaction with the membrane, taking into account that many Gram-positive bacteria contain mostly anionic lipids [34]. The stock solution was prepared in chloroform:methanol $(4: 1 \mathrm{v} / \mathrm{v})$. Surface pressure isotherms were measured in a mini-KSV Langmuir trough (KSV Instruments Ltd, Helsinki, Finland) equipped with a Wilhelmy plate made of filter paper, at $21^{\circ} \mathrm{C}$. The isotherms were obtained by spreading $50 \mu \mathrm{L}$ of the lipid from a stock solution at $627 \mu \mathrm{M}$ on the air/water interface. Prior to the $\pi-\mathrm{A}$ isotherms, adsorption kinetics of the peptide at different concentrations were obtained on the lipid monolayer (at null surface pressure). The surface pressure was monitored with time to ensure adsorption had reached equilibrium before compressing the monolayer. Compression was carried out using two movable barriers at $10 \mathrm{~cm}^{2} \mathrm{~min}^{-1}$. Surface pressure isotherms were performed in triplicate, and the maximum error found was $3 \AA^{2} /$ molecule.

Polarization-Modulated Infrared Reflection-Absorption Spectroscopy (PM-IRRAS) measurements were performed using a KSV PMI 550 instrument (KSV Instruments Ltd, Helsinki, Finland) in a mini KSV Langmuir trough. The light beam reached the monolayer at a fixed incidence angle of $81^{\circ}$, for which the upward-oriented bands indicate a transition moment preferentially parallel the surface plane, whereas downward bands indicate orientation perpendicular to the surface. All the experiments were carried out in a clean room at $21.0 \pm 0.1^{\circ} \mathrm{C}$. The experimental setup was the same used above in Langmuir monolayers. In these PM-IRRAS experiments, we used the highest concentration of each peptide to amplify the band signal. Spectra were collected every $5 \mathrm{mN} / \mathrm{m}$ of surface pressure.

\subsection{Carboxyfluorescein (CF) release from LUVs}

For the leakage assays, LUVs were prepared with S. aureus PL at a concentration of $15 \mathrm{mM}$. The mixture of lipids was dried under a $\mathrm{N}_{2}$ stream and left in vacuum for $6 \mathrm{~h}$ to form a lipid film. First, multilamellar vesicles were obtained by mechanical stirring with a $30 \mathrm{mM}$ HEPES buffer solution, pH 7.4, with $50 \mathrm{mM} \mathrm{CF}$ and $86 \mathrm{mM}$ glucose, added to adjust the solution osmolarity. The suspension was then extruded to get vesicles with uniform size, using a polycarbonate porous membrane to render $100 \mathrm{~nm}$ unilamellar vesicles. This solution was eluted by size-exclusion chromatography through a Sephadex G-50 column to remove the free CF outside the vesicles, using $30 \mathrm{mM}$ HEPES buffer, pH 7.4, with $100 \mathrm{mM} \mathrm{NaCl}$. The CF-LUVs were collected in tubes, diluted and the phospholipid concentration was determined by phosphorous analysis according to the methodology by Rouser et al. [35]. The fluorescence emission of CF was monitored at $\lambda=517 \mathrm{~nm}$, with excitation at $\lambda=492 \mathrm{~nm}$ (slit widths $5 \mathrm{~nm}$ ), using a spectrofluorometer (Cary Eclipse, Varian). Different concentrations of peptide (Lo and $\mathrm{L}_{1}$ ) were added to the CF-LUVs suspension. At the end of each experiment Triton X-100 (1\% v:v) was added for the release of all CF. The percentage of $\mathrm{CF}$ leakage was calculated with the equation: 100 (Ft-Fo)/(Fmax-Fo), where $\mathrm{Ft}$ is the fluorescence at a given time, Fo is the initial fluorescence (before addition of peptide), and Fmax is the maximum fluorescence after addition of Triton X-100 [36-38]. All experiments were performed in triplicate.

Dynamic light scattering (DLS) measurements were performed using a Malvern Zetasizer Nano S equipped with a $633 \mathrm{~nm}$ laser and a detector at $173^{\circ}$ backscattering angle operating at $25^{\circ} \mathrm{C}$. Carboxyfluorescein-entrapped LUVs $(100 \mathrm{~nm}, 1 \mathrm{~mL}, 15 \mathrm{mM})$ prepared with the phospholipid composition of the $S$. aureus was incubated with Lo and $\mathrm{L}_{1}(0.071 \mu \mathrm{M})$ peptides for $10 \mathrm{~min}$. Particle size distributions were analysed using the intensity distribution. All experiments were performed in triplicate.

\section{Results and discussion}

\subsection{Bactericidal activity}

Lo and L1 bactericidal activity were evaluated against S. aureus ATCC 25923. For the cyclic peptide, no bacterial growth was observed, even for concentrations as low as $0.23 \mu \mathrm{M}$, indicating high activity against $S$. aureus. For the linear analogue, L1, the ability to kill S. aureus was observed for concentrations above $28.6 \mu \mathrm{M}$. These data show that Lo is a potent antibiotic against $S$. aureus, with considerably higher activity than its linear analogue L1. In order to understand the importance of the cyclic structure on the bactericidal activity at the molecular level, Langmuir monolayers were used to mimic the bacterial membrane.

\subsection{Surface pressure-area isotherms}

The peptides Lo and $\mathrm{L}_{1}$ adsorbed on a bare air/water interface to form Gibbs films, whose isotherms are shown in Fig. S1 of the Supporting information for an injected concentration of $0.071 \mu \mathrm{M}$ and after waiting $6 \mathrm{~h}$ for the adsorption. The linear nature of $\mathrm{L}_{1}$ leads to a more condensed film, with higher collapse pressure $(25 \mathrm{mN} / \mathrm{m})$ 

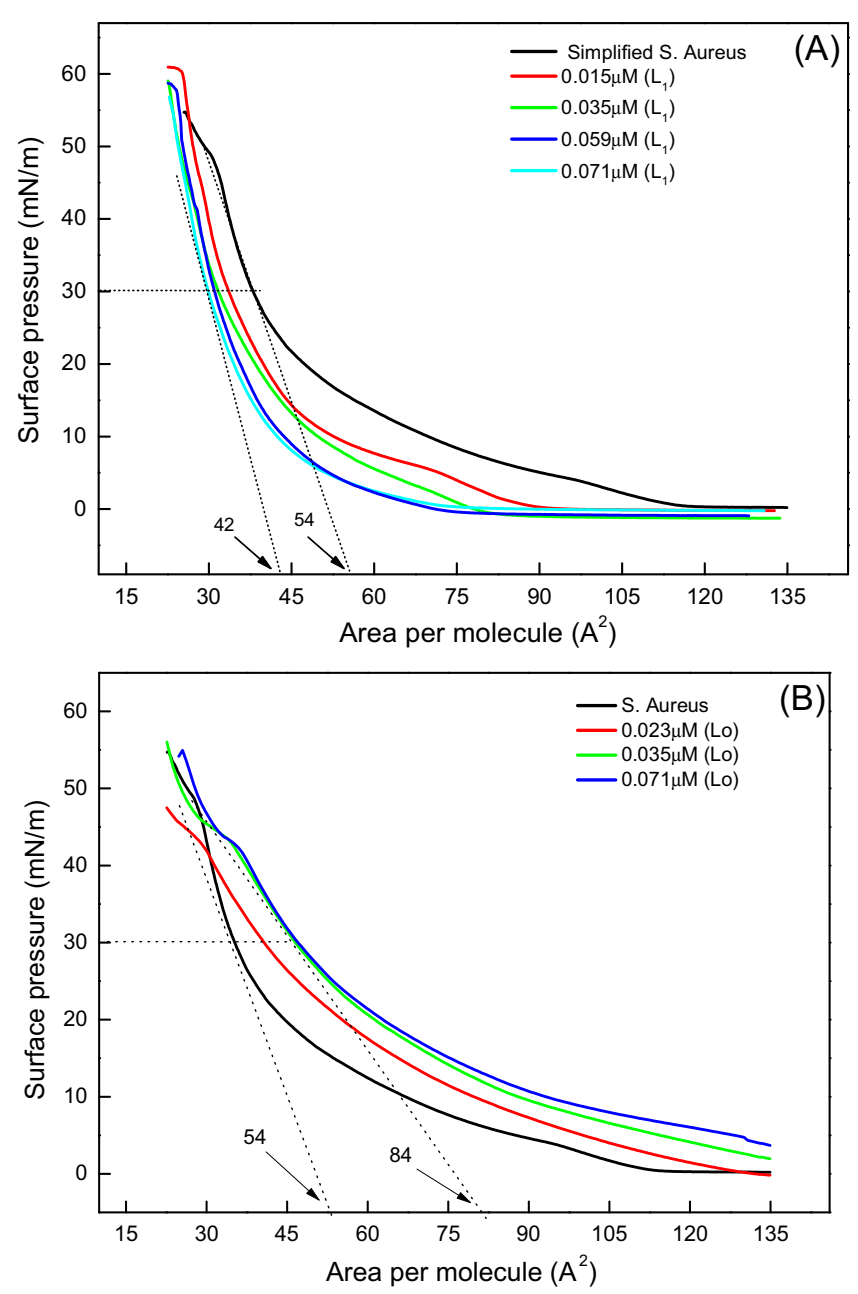

Fig. 1. Surface pressure-area isotherms for monolayers made with the phospholipid composition of $S$. aureus PL, in the absence and presence of $\mathrm{L}_{1}(\mathrm{~A})$ and $\mathrm{Lo}(\mathrm{B})$.

than for the cyclic Lo peptide $(16 \mathrm{mN} / \mathrm{m})$. When made to interact with Langmuir monolayers of 55\% DOPG and 45\% CL representing Gram-positive bacteria [18-20], the peptides exhibited opposite behavior. Fig. 1 shows that while $\mathrm{L}_{1}$ induced a shift to smaller areas per phospholipid, Lo caused the monolayer to expand. The extent of shift caused by $\mathrm{L}_{1}$ increased with concentration up to $0.059 \mu \mathrm{M}$, after which it apparently saturated. Moreover, the plateau in the surface pressure isotherm was gradually extinguished. This shift in area may be explained by either a removal of lipid molecules from the interface, or condensation of the monolayer promoted by the peptide. In contrast, Lo induced a shift to larger areas in the $S$. aureus PL monolayer, which increased with Lo concentration before saturating (Fig. 1B).

The interaction between Lo and $\mathrm{L}_{1}$ with $S$. aureus PL was confirmed in subsidiary experiments to determine the exclusion surface pressure, which serves to indicate whether a guest molecule can adsorb onto a monolayer at distinct surface pressures. Fig. $\mathrm{S} 2$ in the Supporting information shows that both Lo and $\mathrm{L}_{1}$ can be inserted in the monolayer at all high surface pressures tested. Indeed, their interaction with the $S$. aureus PL monolayer was even stronger for high surface pressures, with larger changes in the surface pressure.

\subsection{PM-IRRAS: secondary structure and orientation}

The adsorption of Lo and $\mathrm{L}_{1}$ in Gibbs monolayers could be monitored with PM-IRRAS spectra taken at different time intervals and
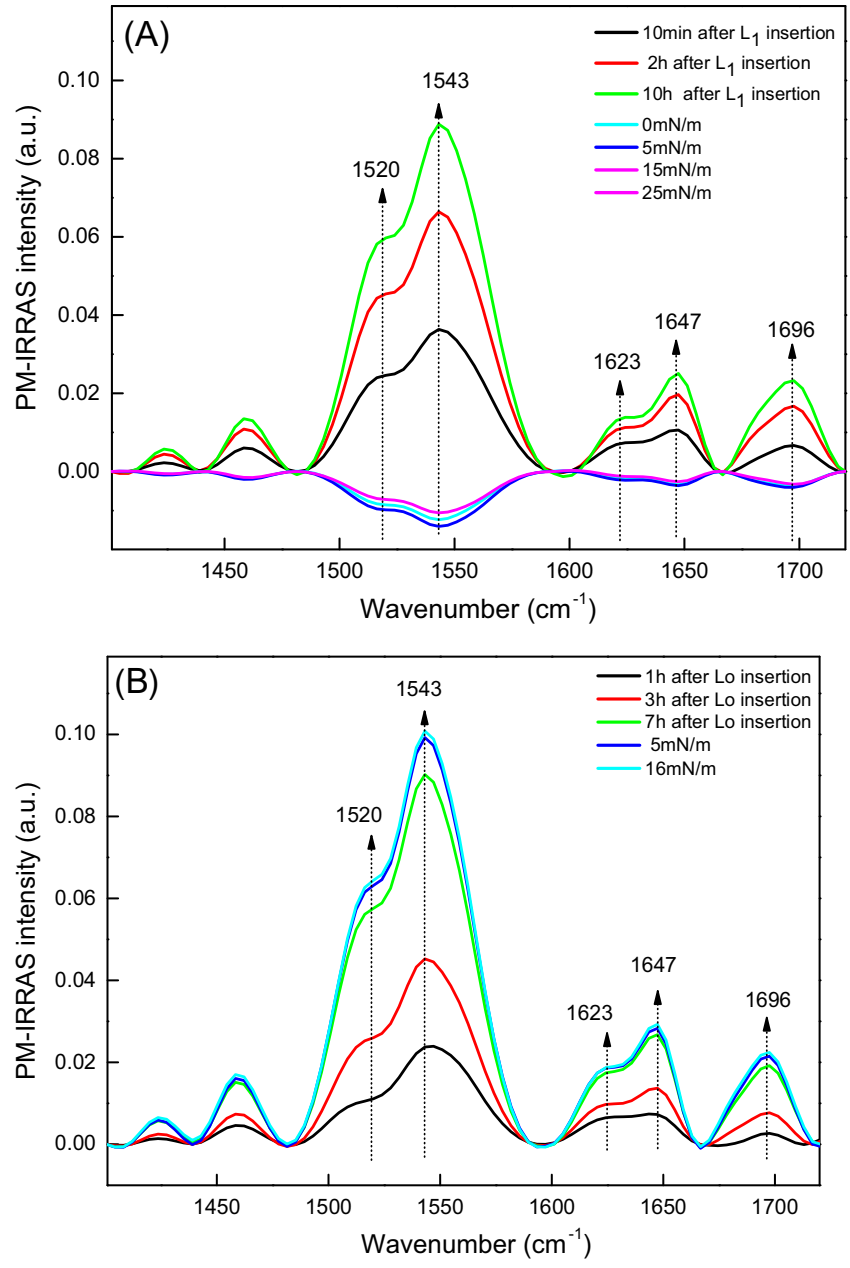

Fig. 2. PM-IRRAS spectra for Gibbs monolayers during adsorption kinetics and then at distinct pressures upon compression, for $\mathrm{L}_{1}(\mathrm{~A})$ and Lo (B) peptides.

then at different surface pressures. Fig. 2A and B show that the typical bands for Lo and $L_{1}$ have their intensity increased with increasing time of adsorption, as one should expect. The bands for the Gibbs monolayers from the linear and cyclic peptides are the same, revealing the same secondary structure for both peptides. Major bands appear at 1623, 1647, and $1696 \mathrm{~cm}^{-1}$ assigned to amide I stretching, and at 1520 and $1543 \mathrm{~cm}^{-1}$, assigned to amide II. The assignment of all bands is given in Table 1 . These bands show predominance of $\beta$-sheets, antiparallel $\beta$-sheets and random coils for the monolayer $[21,22]$. When the Gibbs monolayer for $L_{1}$ was compressed, the bands turned negative, probably because the peptide had its orientation altered in relation to the interface [23]. In contrast, for the cyclic Lo Fig. $2 \mathrm{~B}$ shows that the band intensity increased with the time of adsorption, and then increased even further when the monolayer was compressed. This means that Lo, which is rigid, had its orientation preserved upon compression.

The proportion of each secondary structure in a PM-IRRAS spectrum taken at the amide I region cannot be determined in a straight forward manner by integrating the area under the bands because the intensity depends on the orientation of the group, in addition to their density. Bellet-Amalric et al. [24] developed a method to estimate the percentage of secondary structures by taking the intensity $I_{i}$ of a given band in the PM-IRRAS spectra as proportional to an orientational function $R_{i}(\theta)$ of the transition moment of the amide I associated with the secondary structure $i$, the number $N_{i}$ of the amide I groups being probed in the laser spot with the secondary structure $i$, and the magnitude $M_{i}$ of the transition dipole 
Table 1

PM-IRRAS values $\left(\mathrm{cm}^{-1}\right)$ of the amide I and II regions for pure $S$. aureus phospholipid monolayers in the presence of Lo and $\mathrm{L}_{1}$ peptide (0.071 $\left.\mu \mathrm{M}\right)$, separately.

\begin{tabular}{|c|c|c|c|c|c|c|}
\hline & \multicolumn{3}{|l|}{ Lo } & \multicolumn{3}{|l|}{$\mathrm{L}_{1}$} \\
\hline & Amide I & Amide II & $\begin{array}{l}\text { Secondary structure } \\
\text { composition }\end{array}$ & Amide I & Amide II & $\begin{array}{l}\text { Secondary structure } \\
\text { composition }\end{array}$ \\
\hline Gibbs monolayer & $\begin{array}{l}1696 \\
1647 \\
1623\end{array}$ & $\begin{array}{l}1543 \\
1520\end{array}$ & $\begin{array}{l}\sim 16 \% \text { of } \beta \text {-sheets } \\
\sim 26 \% \text { antiparallel } \\
\sim 58 \% \text { random coil }\end{array}$ & $\begin{array}{l}1696 \\
1647 \\
1623\end{array}$ & $\begin{array}{l}1543 \\
1520\end{array}$ & $\begin{array}{l}\sim 21 \% \beta \text {-sheets } \\
\sim 17 \% \text { antiparallel } \\
\sim 61 \% \text { random coil }\end{array}$ \\
\hline $\begin{array}{l}\text { S. aureus } P L \\
\text { monolayer }\end{array}$ & $\begin{array}{l}1695 \\
1648 \\
1623\end{array}$ & 1545 & $\begin{array}{l}\sim 17 \% \beta \text {-sheets } \\
\sim 23 \% \text { antiparallel } \\
\sim 60 \% \text { random coil }\end{array}$ & 1656 & 1533 & Mostly $\alpha$-Helix \\
\hline
\end{tabular}

moment. That is to say $I_{i} \propto R_{i}(\theta) N_{i} M_{i}$. Often, $M_{i}$ is the same for different structures, as for $\alpha$-helix and $\beta$-sheet conformations [25], then the number of amide I groups with structure $i$ is $N_{i} \propto I_{i} / R_{i}(\theta)$, with the relative percentage being

$P_{i}(\%)=\frac{N_{i}}{\sum N}=\left(\frac{I_{i}}{R_{i}(\theta)} /\right.$

Taking this orientation function in a monolayer of peptides, the flat orientation of $\beta$-sheets $\left(1623 \mathrm{~cm}^{-1}\right)$ and antiparallel $\beta$-sheets $\left(1690 \mathrm{~cm}^{-1}\right)$ lead to the value of 1 . On the other hand, the transition moment of a random coil secondary structure is isotropic, and takes the orientation function equal to 2 . One can thus calculate the intensity of the amide I band corrected by their orientation, using

$I_{1}=\sum_{k} \frac{I_{k}}{R_{k}(\theta)}=I_{1623}+2 I_{1647}+I_{1690}$

and then calculate the proportion of each secondary structure of the Lo and $\mathrm{L}_{1}$ peptides. Upon decomposing the amide $\mathrm{I}$ band of $\mathrm{L}_{1}$, we obtained $\sim 21 \%$ of $\beta$-sheets, $\sim 17 \%$ of antiparallel $\beta$-sheets and $\sim 61 \%$ of random coils. For Lo, we obtained $\sim 16 \%$ of $\beta$-sheets, $\sim 26 \%$ of antiparallel $\beta$-sheets and $\sim 58 \%$ of random coils. Therefore, for the Gibbs monolayers of both peptides there is predominance of random structures, with percentages varying by ca. $\pm 4 \%$ upon changing the surface pressure.

Consistent with the results for the Gibbs monolayers, the amide bands of Lo were barely affected when this peptide was made to interact with a $S$. aureus PL monolayer, as shown in Fig. 3B. The fractions of each secondary structure were kept the same, being dominated by random coils. The band intensity increased with time of adsorption and surface pressure, as it had occurred for the Lo Gibbs monolayer. This result corroborates previous data where the peptide kept its unordered structure even after interacting with LPC and SDS micelles [16], which is due to conformational restrictions of the cyclic peptide. In contrast, Fig. $3 \mathrm{~A}$ shows that the amide bands of $\mathrm{L}_{1}$ were affected by the $S$. aureus PL monolayer (see Table 1 ). Changes are associated with the amide I and II bands at 1656 and $1533 \mathrm{~cm}^{-1}$, respectively, related to the secondary structure of the linear peptide now assuming a major $\alpha$-helix structure upon interacting with S. aureus monolayer. Again, this is consistent with results from the interaction between $\mathrm{L}_{1}$ and LPC and SDS micelles [16], probably ascribed to the peptide linear structure, which allows $\mathrm{L}_{1}$ to adopt a different conformation. Castano and co-workers estimated the orientation of $\alpha$-helix peptides at the interfaces when interacting with monolayers through the ratio of the amide I and II band intensities. Using their model we calculated that the helical axis of $\mathrm{L}_{1}$ is oriented between $53^{\circ}$ and $45^{\circ}$ in relation to the surface plane. Only at the surface pressure of $5 \mathrm{mN} / \mathrm{m}$ was the peptide more upright to ca. $68^{\circ}$, which shows a change in orientation when compression started [26].

The PM-IRRAS technique is also suitable to investigate the packing of lipid chains in Langmuir monolayers, particularly by probing the methylene stretching vibrations. The spectrum for $S$. aureus
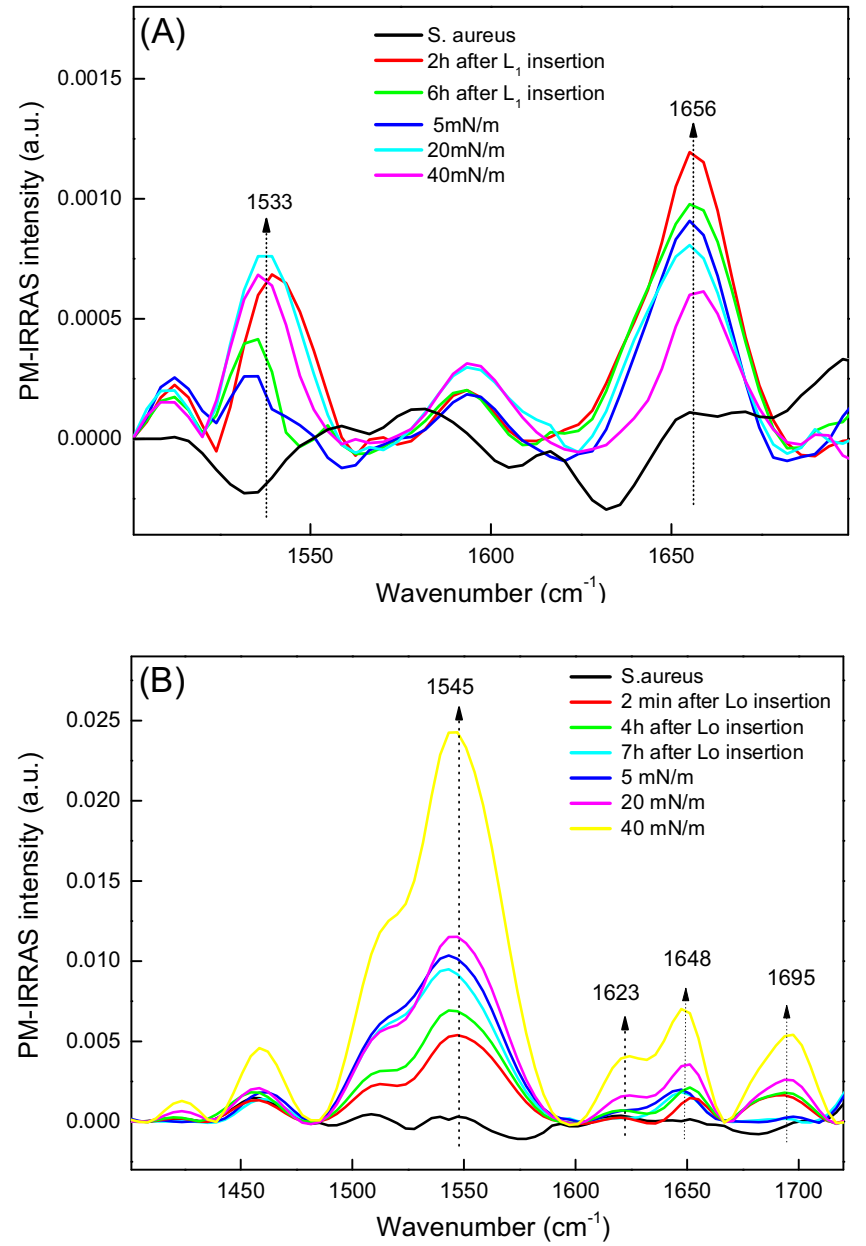

Fig. 3. PM-IRRAS spectra in amide I and II regions for pure $S$. aureus PL composition, and upon the addition of peptide, $\mathrm{L}_{1}$ (A) and Lo (B), during adsorption kinetics and compression.

PL monolayer in Fig. 4 features the asymmetric $\left(\nu_{\mathrm{a}}-\mathrm{CH}_{2}\right)$ and symmetric $\left(\nu_{\mathrm{s}}-\mathrm{CH}_{2}\right)$ methylene stretching vibrations at 2920 and $2850 \mathrm{~cm}^{-1}$, respectively. The appearance of $\nu_{\mathrm{a}}-\mathrm{CH}_{2}$ at $2920 \mathrm{~cm}^{-1}$ is expected and indicates an all-trans state in which the $\mathrm{CH}_{2}$ groups are ordered. We shall concentrate on the more intense $v_{\mathrm{a}}-\mathrm{CH}_{2}$ to identify the effects from the peptides. $\mathrm{L}_{1}$ induced a small shift in the $v_{\mathrm{a}}-\mathrm{CH}_{2}$ band to smaller wavenumbers, reaching $2918 \mathrm{~cm}^{-1}$ at $50 \mathrm{mN} / \mathrm{m}$ (Fig. $4 \mathrm{~A}$ ), owing to a more close packing of the phospholipid chains. In contrast, this band was shifted to larger wavenumbers by Lo, from 2920 to $2925 \mathrm{~cm}^{-1}$ at $50 \mathrm{mN} / \mathrm{m}$ (Fig. 4B). The phospholipids became less ordered since the cyclic Lo should occupy a larger area, as indicated in the surface pressure isotherms.

Therefore, both peptides (Lo and $\mathrm{L}_{1}$ ) interacted with the monolayer (Table 1 ), but their mechanisms of action differ. $\mathrm{L}_{1}$ caused 

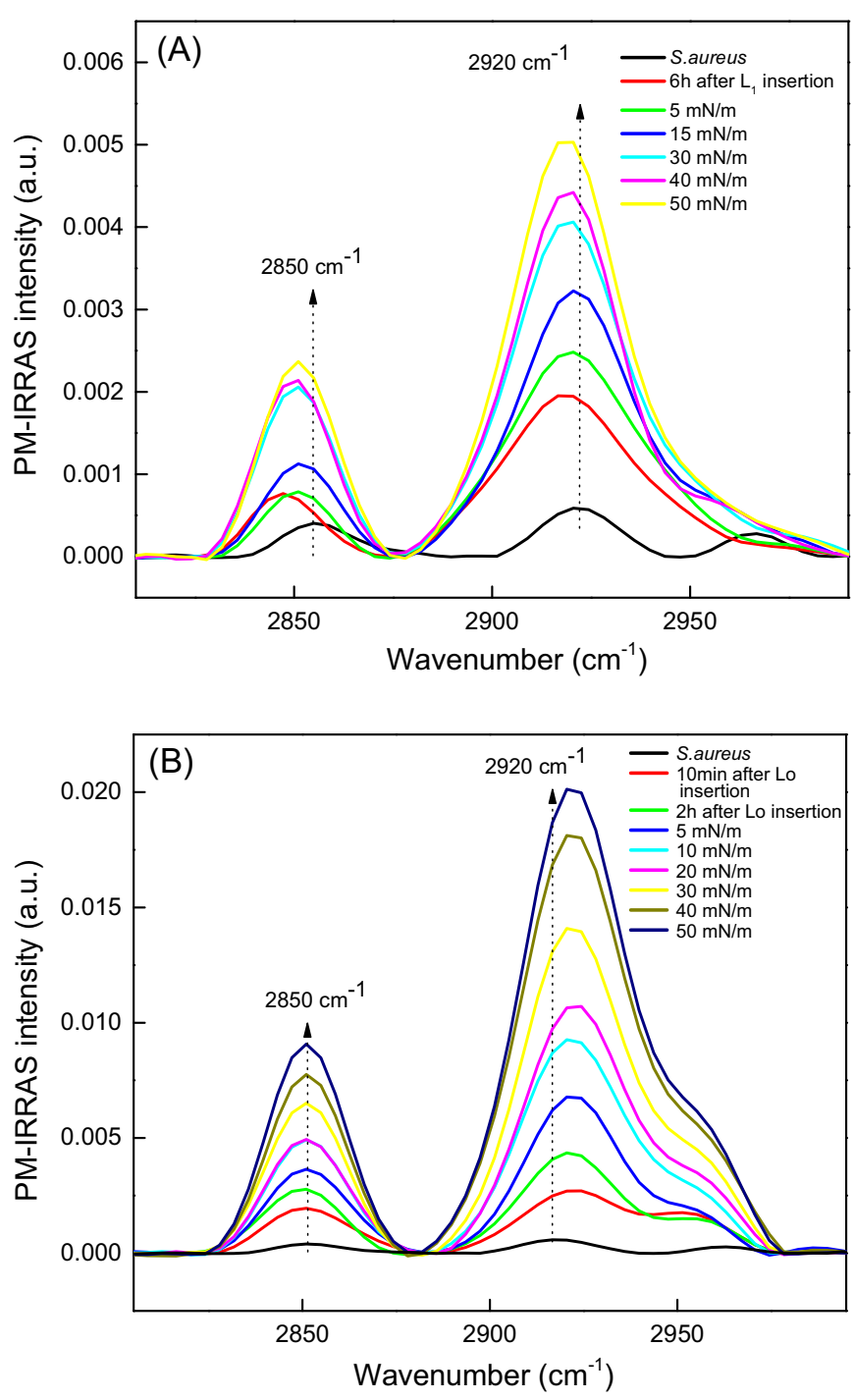

Fig. 4. PM-IRRAS spectra in the region of the methylene stretching bands (2800-3000 $\mathrm{cm}^{-1}$ ) for pure S. aureus PL monolayer (-) spread on water subphase, and upon the addition of $\mathrm{L}_{1}(\mathrm{~A})$ and $\mathrm{Lo}(\mathrm{B})$ peptides.

compaction of the lipid monolayer, thus inhibiting insertion of further $\mathrm{L}_{1}$ molecules. Furthermore, at high surface pressures its conformation changed, which has an impact on the mechanism of action. In contrast, Lo caused the membrane to expand and become more fluid, and its conformation did not change, which is important for its biological activity against microbes. Taken together, the data from monolayers brought useful information about the interaction of each peptide with $S$. aureus PL. It was clear that Lo and $\mathrm{L}_{1}$ interacted in a very different manner with the membrane, which propably explains their differences in bactericidal action. Also, with PM-IRRAS we could obtain information about the peptideís secondary structure, which usually plays an important role on their mechanism of action. However, the data so far presented are not sufficient to identify the mechanism of action of Lo peptide and explain why $\mathrm{L}_{1}$ is not so efficient in killing bacteria.

It is worth discussing the possible types of interaction responsible for the effects. Though the present study was conducted only with negatively charged phospholipids (which are the major constituents of S. aureus membrane), it is possible to conclude that hydrophobic interactions are the driving force for the peptide interaction with the membrane. This inference can be made based on previous data for Labaditin interacting with LPC and SDS micelles

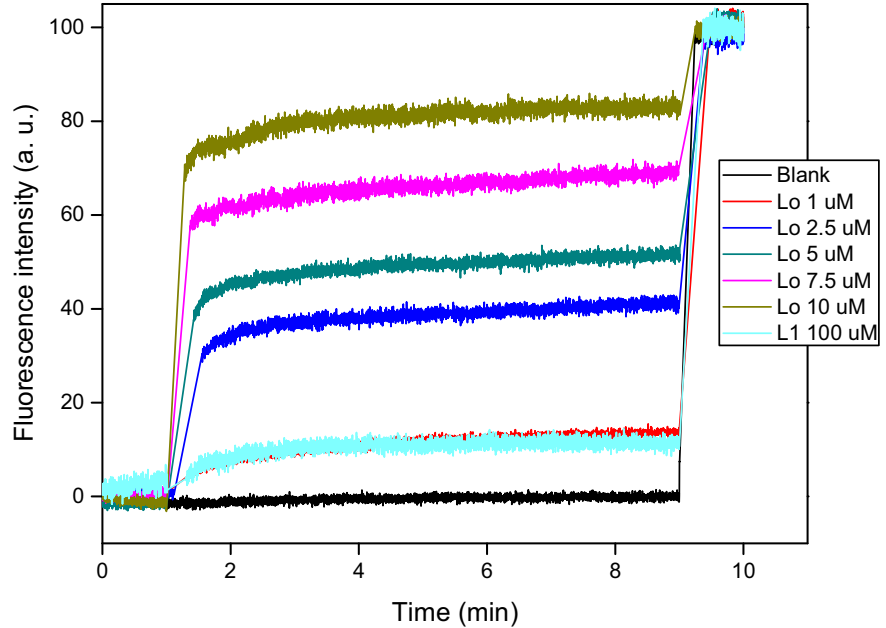

Fig. 5. Kinetics of CF leakage from LUVs made of $S$. aureus $P L$ in response to addition of different concentrations of $\mathrm{L}_{1}$ and Lo peptides. The peptides were injected $1 \mathrm{~min}$ after the beginning of the kinetics measurements. After 9 min, Triton X-100 (1\%) was added to induce complete leakage of CF.

[16]. In the latter study, data from fluorescence emission, values of the Stern-Volmer constant and molecular dynamics simulations indicated that the peptide was able to interact with anionic and zwitterionic micelles. Therefore, the peptide structure appears more relevant than the charge of the membrane.

\subsection{Membrane permeability assay}

Most AMPs alter the microbial membrane and therefore affect its integrity, either by forming pores or solubilizing the membrane via the 'carpet' mechanism, thus yielding some membrane permeability $[7,27]$. In order to investigate whether Lo and $\mathrm{L}_{1}$ could permeate the membrane, LUVs were produced from $S$. aureus PL containing the $\mathrm{CF}$ fluorescent dye encapsulated. A low intensity fluorescence was measured before addition of the peptides due to the self-quenching of $\mathrm{CF}$, and this was taken as the control value for $0 \%$ of leakage (blank) (Fig. 5). $\mathrm{L}_{1}$ was not able to cause significant leakage even at a concentration of $100 \mu \mathrm{M}$. With these results the hypothesis of removal of phospholipids from the interface, which would explain the shift to smaller molecular areas on the surface pressure isotherms for $S$. aureus PL, is discarded. It is thus concluded that $\mathrm{L}_{1}$ was incorporated and altered the packing of $S$. aureus PL molecules to be more closely packed (both in Langmuir monolayers and LUVs). In contrast, Lo induced a concentration-dependent leakage, ranging from 13 to $84 \%$ for concentrations in the range between 1 and $10 \mu \mathrm{M}$. This concentration dependence can be explained in terms of a pore forming mechanism [28].

The ability to induce leakage in LUVs by Lo but not by $\mathrm{L}_{1}$ explains why Lo had a bactericide effect while $\mathrm{L}_{1}$ did not. These results are similar to those for the cyclic broad-spectrum antimicrobial peptide, Tachyplesin I. Linear analogues of Tachyplesin I, synthesized by protecting SH groups or replacing cysteins by alanines to avoid cyclization, were not able to kill bacteria. In addition, Tachyplesin I was able to induce leakage of calcein from LUVs made of phosphatidylglycerol (PG), but not from phosphatidylcholine LUVs [11], and therefore the membrane charge is important for the peptide to develop its action $[12,29]$. Although the correlation between cyclic structure and bactericidal activity seems to be confirmed for these peptides, a detailed mechanism that explains how these molecules are able to act is still unclear.

Pore formation by AMPs has been well studied, mainly for linear molecules. It promotes leakage of internal fluids, killing the cells. This pore is usually formed by insertion of a peptide into the 

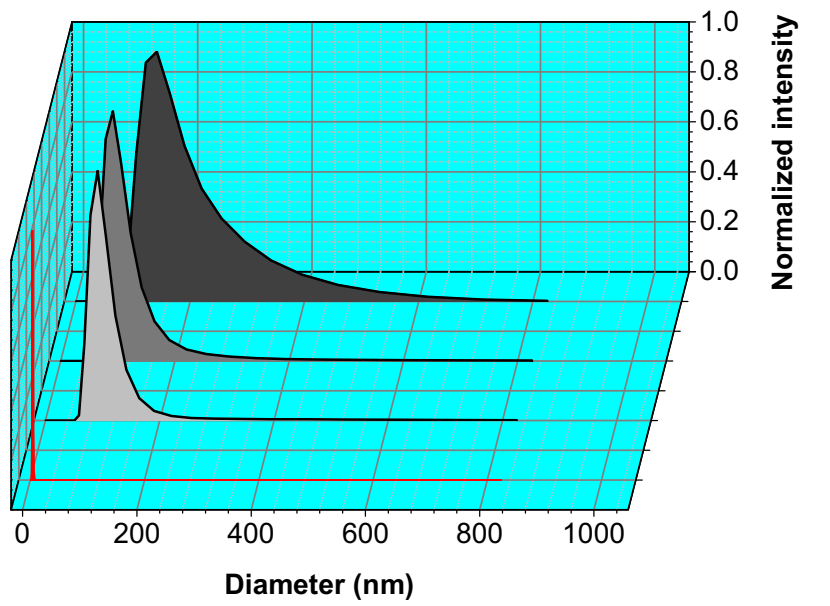

Fig. 6. Analysis of diameter distributions by Dynamic Light Scattering obtained with pure S. aureus phospholipids LUVs $\left(\boldsymbol{\square}^{-}\right)$, and upon the addition of Lo $\left(^{\square}\right), \mathrm{L}_{1}\left({ }^{\square}\right)$, or triton X-100( $\left.{ }^{\square}\right)$.

membrane forming a barrel-stave or a toroidal pore [7]. However, to form a pore the peptide has to be able to cross the membrane. Peptides that form $\alpha$-helices shorter than the bilayer thickness, which is probably the case of the linear analogue of Labaditin $\left(\mathrm{L}_{1}\right)$, are not able to kill the bacteria. Having this in mind, we speculate whether Labaditin (Lo) is able to traverse the membrane, since this peptide has structural restrictions due to its cyclic nature. A second option is that the peptide could partially solubilize the membrane by interacting and removing part of the lipid molecules. This can in principle be learnt by testing whether LUVs are destroyed by interacting with the peptides, for which DLS experiments are useful.

\subsection{DLS measurements}

DLS measurements were performed to verify the integrity of the vesicles after their interaction with the peptides. Fig. 6 shows the size distribution profile for the vesicles in absence and presence of the peptides, as well as after treatment with $1 \%$ of Triton X-100. The DLS experiments were performed using the same LUVs preparation for the permeabilizaton assays.

According to Fig. 6, the addition of Lo and $\mathrm{L}_{1}$ did not alter significantly the hydrodynamic radius of the vesicles. For Lo, the LUVs had their integrity preserved during the leakage process, i.e. leakage did not result in vesicle destruction. The presence of the LUVs was also confirmed by addition of Triton X-100 to their suspension, which caused vesicle disruption due to membrane solubilization. For all samples the polydispersity index was smaller than 0.1, i.e. the solution was monodisperse. From the DLS data we may discard the possibility of membrane solubilisation by Lo as a major mechanism of action. Indeed, the polydispersity decreased in the presence of peptides, and the majority of the vesicles kept their original size. The inevitable conclusion is that Lo acts by forming pores, maintaining the integrity of LUVs structure but allowing the leakage of internal cell components through the membrane. In a study with a series of cyclic peptides, Danial and coworkers [30] observed that cyclic peptides with neutral side chains, such as Labaditin, are able to form unimeric pores by stacking of peptide units until reaching the membrane thickness. Since Lo is a small, cyclic peptide, the most probable mechanism for pore formation is the self-assembly of Lo molecules in such a way that it could cross the membrane. This assembly results in structures referred to as peptide nanotubes $[31,32]$, a recent and promising class of antibiotics with high specificity toward bacterial cells, without being harmful to mammalian cells.

\section{Conclusions}

The cyclic peptide Labaditin (Lo) was proven as highly efficient in killing $S$. aureus, in contrast to its linear analogue $\left(\mathrm{L}_{1}\right)$, and this difference in activity was explained here by their different effects on model cell membranes. The membrane of S. aureus, mimicked with a Langmuir monolayer obtained with a phospholipid mixture containing 55\% DOPG and $45 \% \mathrm{CL}$, interacted with both Lo and $\mathrm{L}_{1}$, but the mode of interaction differed. Lo induced an increase in the area per molecule, which was opposite to the effect of $L_{1}$. Most importantly, the PM-IRRAS data indicated that the conformation of Lo was not affected by the interaction with the monolayer, but the secondary structure of $\mathrm{L}_{1}$ changed from random coils mainly to $\alpha$-helix structures. Therefore the conformational restriction of Lo appears to be the key property for the effect on the cell membrane model responsible for its bactericidal activity.

With assays of membrane permeability, we found that Lo but not $\mathrm{L}_{1}$ - induces leakage in LUVs, via formation of pores. The hypothesis of vesicle destruction was discarded based on dynamic light scattering experiments. As Lo is a small, cyclic peptide, pore formation can only take place if several such molecules self-assemble to yield a nanotube.

In summary, the bactericidal activity of Lo is attributed to its inducing leakage in the cell membrane, and thus it does not depend on the presence of specific metabolic targets. Leakage occurs with the cyclic Lo self-assembling into a nanotube owing to its preserved secondary structure in the membrane. This mode of action may represent a promising step toward the design of a new class of antibiotics for which bacteria resistance will not be developed, at least not via the conventional routes.

\section{Acknowledgments}

The authors are grateful to CNPq, FAPESP (2014/03748-9), CAPES and nBioNet network (Brazil) for financial support.

\section{Appendix A. Supplementary data}

Supplementary data associated with this article can be found, in the online version, at http://dx.doi.org/10.1016/j.colsurfb.2016.09. 017.

\section{References}

[1] F. Devapriya, R. Ramesh, A.K. Sajit Khan, J. Shanmugam, $\beta$-Lactamase production of Staphylococcus aureus: a comparison study of different iodometric methods, Gulf Med. J. 2 (2013) 6.

[2] C.K. Naber, Staphylococcus aureus bacteremia: epidemiology, pathophysiology, and management strategies, Clin. Infect. Dis. 48 (Suppl. 4) (2009) S231-237.

[3] J.P. da Costa, M. Cova, R. Ferreira, R. Vitorino, Antimicrobial peptides: an alternative for innovative medicines? Appl. Microbiol. Biotechnol. 99 (2015) 2023-2040.

[4] C.D. Fjell, J.A. Hiss, R.E. Hancock, G. Schneider, Designing antimicrobial peptides: form follows function, Nat. Rev. Drug Discov. 11 (2012) 37-51.

[5] R.E. Hancock, Cationic peptides: effectors in innate immunity and novel antimicrobials, Lancet Infect. Dis. 1 (2001) 156-164.

[6] G. Roscia, C. Falciani, L. Bracci, A. Pini, The development of antimicrobial peptides as new antibacterial drugs, Curr. Protein Pept. Sci. 14 (2013) 641-649.

[7] K.A. Brogden, Antimicrobial peptides: pore formers or metabolic inhibitors in bacteria? Nat. Rev. Microbiol. 3 (2005) 238-250.

[8] H. Khandelia, J.H. Ipsen, O.G. Mouritsen, The impact of peptides on lipid membranes, Biochim. Biophys. Acta 1778 (2008) 1528-1536.

[9] B. Mishra, G. Wang, The importance of amino acid composition in natural AMPs: an evolutional structural, and functional perspective, Front. Immunol. 3 (2012) 221.

[10] M.A. Sani, T.C. Whitwell, F. Separovic, Lipid composition regulates the conformation and insertion of the antimicrobial peptide maculatin 1.1, Biochim. Biophys. Acta 1818 (2012) 205-211.

[11] K. Matsuzaki, M. Fukui, N. Fujii, K. Miyajima, Interactions of an antimicrobial peptide tachyplesin I, with lipid membranes, Biochim. Biophys. Acta 1070 (1991) 259-264 
[12] K. Matsuzaki, Magainins as paradigm for the mode of action of pore forming polypeptides, Biochim. Biophys. Acta (BBA) - Rev. Biomembr. 1376 (1998) 391-400.

[13] A.D. Cirac, G. Moiset, J.T. Mika, A. Kocer, P. Salvador, B. Poolman, S.J. Marrink, D. Sengupta, The molecular basis for antimicrobial activity of pore-forming cyclic peptides, Biophys. J. 100 (2011) 2422-2431.

[14] Q. Xiao, D. Pei, High-throughput synthesis and screening of cyclic peptide antibiotics, J. Med. Chem. 50 (2007) 3132-3137.

[15] A. Roxin, G. Zheng, Flexible or fixed: a comparative review of linear and cyclic cancer-targeting peptides, Future Med. Chem. 4 (2012) 1601-1618.

[16] S.C. Barbosa, E.M. Cilli, L.G. Dias, C.A. Fuzo, L. Degreve, R.G. Stabeli, R. Itri, P. Ciancaglini, Interaction of cyclic and linear Labaditin peptides with anionic and zwitterionic micelles, J. Colloid Interface Sci. 438 (2015) 39-46.

[17] S.C. Barbosa, E.M. Cilli, L.G. Dias, R.G. Stabeli, P. Ciancaglini, Labaditin, a cyclic peptide with rich biotechnological potential: preliminary toxicological studies and structural changes in water and lipid membrane environment, Amino Acids 40 (2011) 135-144.

[18] G.H. Joyce, R.K. Hammond, D.C. White, Changes in membrane lipid composition in exponentially growing Staphylococcus aureus during the shift from 37 to 25 C, J. Bacteriol. 104 (1970) 323-330.

[19] P.R. Beining, E. Huff, B. Prescott, T.S. Theodore, Characterization of the lipids of mesosomal vesicles and plasma membranes from Staphylococcus aureus, J. Bacteriol. 121 (1975) 137-143.

[20] R.F. Epand, P.B. Savage, R.M. Epand, Bacterial lipid composition and the antimicrobial efficacy of cationic steroid compounds (Ceragenins), Biochim. Biophys. Acta 1768 (2007) 2500-2509.

[21] E. Maltseva, A. Kerth, A. Blume, H. Mohwald, G. Brezesinski, Adsorption of amyloid beta (1-40) peptide at phospholipid monolayers, Chembiochem 6 (2005) 1817-1824.

[22] A. Banc, B. Desbat, D. Renard, Y. Popineau, C. Mangavel, L. Navailles, Structure and orientation changes of omega- and gamma-gliadins at the air-water interface: a PM-IRRAS spectroscopy and Brewster angle microscopy study, Langmuir 23 (2007) 13066-13075.

[23] M. Lhor, S.C. Bernier, H. Horchani, S. Bussieres, L. Cantin, B. Desbat, C. Salesse, Comparison between the behavior of different hydrophobic peptides allowing membrane anchoring of proteins, Adv. Colloid Interface Sci. 207 (2014) 223-239.

[24] E. Bellet-Amalric, D. Blaudez, B. Desbat, F. Graner, F. Gauthier, A. Renault, Interaction of the third helix of Antennapedia homeodomain and a phospholipid monolayer, studied by ellipsometry and PM-IRRAS at the air-water interface, Biochim. Biophys. Acta 1467 (2000) 131-143.
[25] T. Buffeteau, E. Le Calvez, S. Castano, B. Desbat, D. Blaudez, J. Dufourcq, Anisotropic optical constants of $\alpha$-helix and $\beta$-sheet secondary structures in the infrared, J. Phys. Chem. B 104 (2000) 4537-4544.

[26] S. Castano, B. Desbat, M. Laguerre, J. Dufourcq, Structure, orientation and affinity for interfaces and lipids of ideally amphipathic lytic $\operatorname{LiKj}(\mathrm{i}=2 \mathrm{j})$ peptides, Biochim. Biophys. Acta 1416 (1999) 176-194.

[27] M.R. Yeaman, N.Y. Yount, Mechanisms of antimicrobial peptide action and resistance, Pharmacol. Rev. 55 (2003) 27-55.

[28] A.L. Russell, A.M. Kennedy, A.M. Spuches, D. Venugopal, J.B. Bhonsle, R.P. Hicks, Spectroscopic and thermodynamic evidence for antimicrobial peptide membrane selectivity, Chem. Phys. Lipids 163 (2010) 488-497.

[29] H. Tamamura, M. Kuroda, M. Masuda, A. Otaka, S. Funakoshi, H. Nakashima, N. Yamamoto, M. Waki, A. Matsumoto, J.M. Lancelin, et al., A comparative study of the solution structures of tachyplesin I and a novel anti-HIV synthetic peptide T22 ([Tyr5,12, Lys7]-polyphemusin II), determined by nuclear magnetic resonance, Biochim. Biophys. Acta 1163 (1993) 209-216.

[30] M. Danial, S. Perrier, K.A. Jolliffe, Effect of the amino acid composition of cyclic peptides on their self-assembly in lipid bilayers, Org. Biomol. Chem. 13 (2015) 2464-2473.

[31] M.R. Ghadiri, J.R. Granja, R.A. Milligan, D.E. McRee, N. Khazanovich, Self-assembling organic nanotubes based on a cyclic peptide architecture, Nature 366 (1993) 324-327.

[32] J.D. Hartgerink, J.R. Granja, R.A. Milligan, M.R. Ghadiri, Self-assembling peptide nanotubes, J. Am. Chem. Soc. 118 (1996) 43-50.

[33] N.J. Mitchell, P. Seaton, A. Pokorny, Branched phospholipids render lipid vesicles more susceptible to membrane-active peptides, Biochim. Biophys. Acta 1858 (2016) 988-994.

[34] R.F. Epand, A. Mor, R.M. Epand, Lipid complexes with cationic peptides and OAKs; their role in antimicrobial action and in the delivery of antimicrobial agents, Cell. Mol. Life Sci. 68 (2011) 2177-2188.

[35] G. Rouser, S. Fleischer, A. Yamamoto, Two dimensional thin layer chromatographic separation of polar lipids and determination of phospholipids by phosphorus analysis of spots, Lipids 5 (1970) 494-496

[36] E.N. Lorenzon, P.R. Sanches, L.G. Nogueira, T.M. Bauab, E.M. Cilli, Dimerization of aurein 1.2: effects in structure, antimicrobial activity and aggregation of Candida albicans cells, Amino Acids 44 (2013) 1521-1528.

[37] A.D. Paiva, N. Irving, E. Breukink, H.C. Mantovani, Interaction with lipid II induces conformational changes in bovicin HC5 structure, Antimicrob. Agents Chemother. 56 (2012) 4586-4593.

[38] B. Mattei, A. Miranda, K.R. Perez, K.A. Riske, Structure-activity relationship of the antimicrobial peptide gomesin: the role of peptide hydrophobicity in its interaction with model membranes, Langmuir 30 (2014) 3513-3521. 